\title{
A forest in a city Biodiversity at Sunut forest, Khartoum, Sudan
}

\section{Huyam Eltayeb, Eihab Idris, Amal Adam, Tasabeeh Ezaldeen \& Dawi Hamed}

Faculty of Science, University of Khartoum., Sudan

E-mail: eihabidriss@gmail.com

\section{ABSTRACT}

Al-Sunut is a natural forest located in the centre of Khartoum state, the capital of Sudan. Due to its unique location, Sunut forest is severely stressed by human activities, especially construction. In this paper, we provide a survey for the biodiversity at Sunut Forest. Three animal groups were investigated for density and species diversity: Birds, Acacia nilotica associated arthropods and ground arthropods. The aim of the study was to provide the field data required for the design of adequate management and conservation plans and to draw the popular attention to the special value of Sunut forest. The study concluded that Sunut forest hosts considerable biological diversity, particularly of birds, as 87 species were recorded. Arthropod specimens that belong to 10 different orders were collected from A. nilotica trees. Likewise, specimens of ground arthropods collected from the forest represent 11 different orders.

Keywords: Habitat conservation, Birds, tree-associated arthropods, ground arthropods, Acacia nilotica, Khartoum

\section{INTRODUCTION}

Al-Sunut is a natural forest which borders the White Nile at the Mogran area, Khartoum. Sunut forest is a unique biotope; a poor savannah habitat impeded in the semi desert background of Northern Sudan (Shawki \& Musnad, 1964). As a consequence, the forest is characterized by high density of plant cover, mainly composed of Acacia nilotica trees (Mohamed, 1986; Ahmed, 1998). Moreover, it represents a hot spot of bird and invertebrate diversity. It has been estimated that the forest contains at least 70 bird species (among which 26 are migrants) together with a diverse invertebrate community (Cloudsely-Thompson, 1964; Nikolaus, 1987; Elobeid, 1990; Lado, 1994; Abd-Alrahman, 1998). The major environmental factor which underlies the unusual biodiversity at Sunut forest is the annual floods of the Nile River, which supplies the site with immense amounts of water and nutrient-reach sediments. Thus, the Sunut forest is considered as an inland wetland ecosystem (Altayeb \& Hamed, 2003).

Because of its unique position as a natural forest in the heart of the modern, crowded and growing capital of Sudan, Sunut forest provides a whole range of valuable services to the environment and the society of Khartoum. It is an important ground for both resident and migrating birds. It also acts as a barrier that protects the residential areas in the vicinity from the annual flooding by holding the excess water of the White Nile. Moreover, the local community exploits the forest for fishing, grazing, farming as well as recreation activities. Sunut forest is an attractive touristic site particularly for bird watchers. Because of its accessibility and proximity to academic institutions, Sunut forest was a major part in the field training of generations of Sudanese biologists (Altayeb \& Hamed, 2003). 
The Sunut forest is a national protected area. It is regarded as a forest reserve since 1932 and declared as a bird sanctuary since 1945 . The forest attracted considerable attention at the international level as well. The management category of the International Union for the Conservation of Nature (IUCN) has listed the site as a bird sanctuary. In addition, it has been proposed as a Ramsar site (i.e. wetland of international importance) based on Ramsar convention (Altayeb \& Hamed, 2003). Despite all these efforts, the level of protection that is actually applied at the site remains limited.

Being in the middle of the urban Khartoum region is the reason why Sunut forest is special in the first place, however, it is also the reason why it is extremely vulnerable to human disturbance. The forest is stressed by deforestation, habitat loss, overgrazing and waste disposal (Abushama, 1994). The most important threat, however, is the reclamation of the forest land for construction purposes; for example, about $15 \%$ of the forest area has been removed for the construction of the new White Nile Bridge (Altayeb \& Hamed, 2003). As the Khartoum population continues to grow, it is expected that the threats facing the forest will increase in magnitude, unless adequate management plans are designed and thoroughly implemented.

In this paper, we provide a preliminary investigation of the biodiversity at Sunut forest, based on assessment of three key animal groups: birds, ground arthropods and Acacia nilotica associated arthropods. The goal is to provide a solid base of knowledge in order to guide management and conservation efforts taking place at Sunut forest.

\section{MATERIAL AND METHODS}

\section{Study area}

This study was carried out in the Sunut Forest $\left(15^{\circ} 35^{\prime} \mathrm{N}, 32^{\circ} 30^{\prime} \mathrm{E}\right)$ (Figure 1). The site is bordered by the new White Nile Bridge from the North, the industrial area from the South, the Ghaba Street from the East and the White Nile from the West. Along the river bank there is a narrow cultivated area extending between the forest and the river bank (Figure 2).

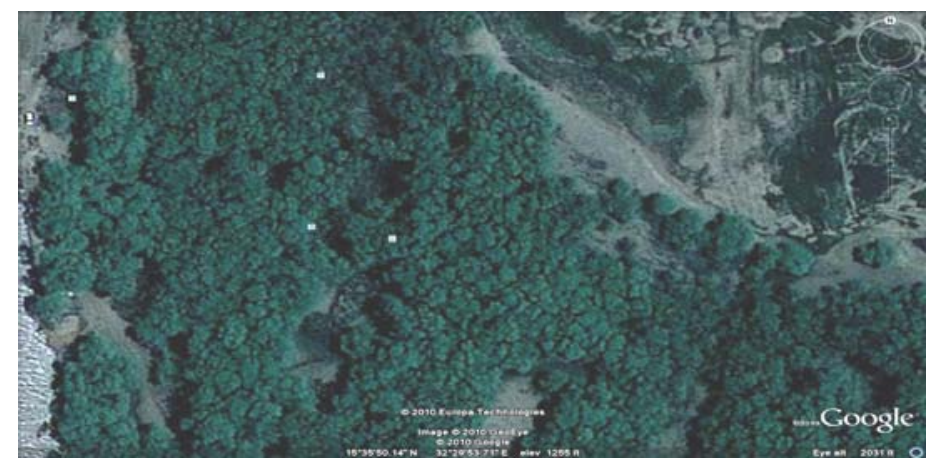

Fig. 1: The Sunut Forest (Satellite image).

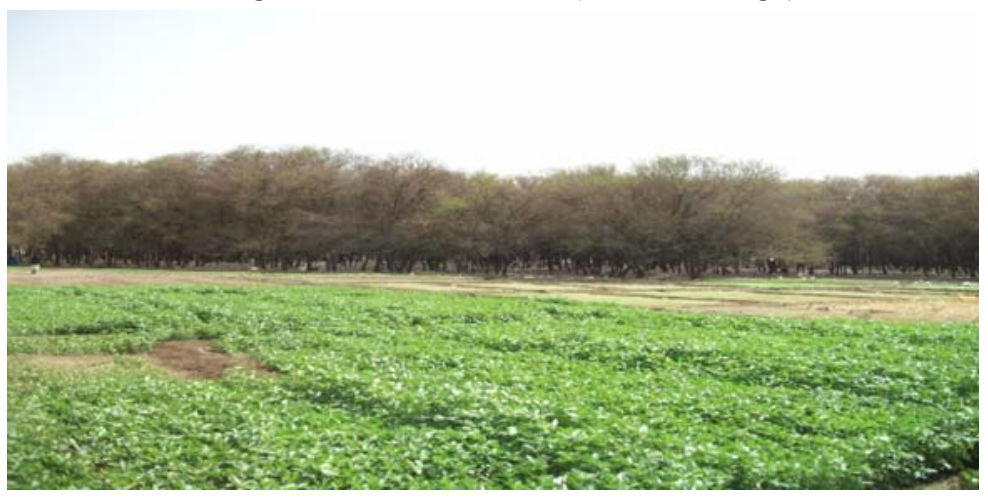

Fig. 2: The cultivated area at the edge of the Sunut forest. 


\section{Climate}

The climate of Khartoum is tropical desert. There are three seasons per year, cool winter, dry summer and a rainy season. Highest temperatures $\left(45^{\circ} \mathrm{C}\right.$ or more $)$ are recorded in summer months (May-June) while lowest temperatures $\left(22^{\circ} \mathrm{C}\right.$ or less) are recorded during winter months (December-January). The rainfall is about $150 \mathrm{~mm}$ per annum. The forest is flooded during the rainy season (July-October). Later, during winter and early summer, the forest becomes totally dry (Eltayeb \& Hamed, 2003).

\section{Investigation of bird diversity}

The survey was carried out between October and June. Assessment of avifauna was based on bird watching. At least one visit was conducted per each month. Observation was performed during the early morning (6:45 am to 11:00 Am). Identification of bird species was made by sight using Binoculars following Stevenson \& Fanshawe (2002). The numbers of individuals observed were counted when numbers were limited or estimated when numbers were huge.

\section{Investigation of Acacia nilotica-associated arthropods}

The survey was conducted in April. Based on field observations, four sampling stations were established. These are located along a transect crossing the forest from the West (River bank) to East (Ghaba street). The four stations were visited for sampling 12 times during the period of April-June 2003. Invertebrate samples were collected from $A$. nilotica trees by beating the trees with a stick and collecting organisms that fall on the beating sheet. Using the aspirator, samples were then drawn into a labeled vial containing $2 \mathrm{ml}$ of ethyl alcohol (70\%). At each station 20 trees were sampled.

\section{Investigation of ground arthropods}

The survey was conducted in February. Ground arthropods were sampled from two different sites in Sunut forest. The first site is located at the cultivated area bordering the Nile while the second area is located about $50 \mathrm{~m}$ from the edge of the forest, away from the river. Specimens were collected using pit fall traps. Traps were filled with water and oil and were set in burrows so that the top of the trap was leveled with the ground surface. Within each site, traps were distributed along three transects, separated by a space of $15 \mathrm{~m}$ between every two traps. Traps were left overnight and visited daily for seven consecutive days. Specimens were collected using forceps and a sieve and were put in labeled bottles containing 70\% ethanol.

\section{Identification of arthropod specimens}

Collected arthropod specimens were brought to the lab, where they were stored, identified to the lowest possible taxa and counted to estimate their density. Identification and counting were conducted under a dissecting microscope and/or a stereomicroscope. Identification was made following the literature. Specimens were sorted to morphospecies, according to size, shape and colour.

\section{RESULTS}

\section{Bird diversity}

Overall, 87 bird species were recorded in this study. Among the recorded species, 50 were Palaearctic migrants, 8 were local migrants and 29 species were resident. Species that were found in the study are listed in table (1). 
Table 1: Bird species observed in the study area: where $\mathrm{R}=$ resident (present throughout the year), $\mathrm{LM}=$ local migrant (undergoing distinct seasonal movements within its distribution), $\mathrm{PM}=$ Palaearctic migrant (non-breeding visitors from the Palaearctic).

\begin{tabular}{|c|c|c|c|c|}
\hline No. & Species Common Name & Status & Scientific Name & Family Name \\
\hline 1 & Great White Pelican & PM & Pelecanus onocrotalus & Pelecanidae \\
\hline 2 & Pink backed Pelican & $\mathrm{R}$ & Pelecanus rufescens & Pelecanidae \\
\hline 3 & Little Grebe & LM & Tachybaptus ruficollis & Podicipedidae \\
\hline 4 & Long-tailed Cormorant & LM & Phalacrocorax africanus & Phalacrocoracidaee \\
\hline 5 & Cattle Egret & PM & Bubulcus ibis & Ardeidae \\
\hline 6 & Common Saquacco Heron & PM & Ardeola ralloides & Ardeidae \\
\hline 7 & Little Egret & PM & Egretta garzetta & Ardeidae \\
\hline 8 & Great Egret & PM & Casmerodius albus & Ardeidae \\
\hline 9 & Goliath Heron & $\mathrm{R}$ & Ardea goliath & Ardeidae \\
\hline 10 & Grey Heron & PM & Ardea cinerea & Ardeidae \\
\hline 11 & White Stork & PM & Ciconia ciconia & Ciconiidae \\
\hline 12 & Abdims Stork & $\mathrm{R}$ & Ciconia abdimii & Ciconiidae \\
\hline 13 & Black Stork & PM & Ciconia nigra & Ciconiidae \\
\hline 14 & Saddle-billed Stork & $\mathrm{R}$ & Ephippiorhynchus senegalensis & Ciconiidae \\
\hline 15 & Sacred Ibis & LM & Threkiornis aethiopicus & Threskiornithidae \\
\hline 16 & African Spoonbill & LM & Platalea alba & Threskiornithidae \\
\hline 17 & Eurasian Spoonbill & PM & Platalea leucorodia & Threskiornithidae \\
\hline 18 & Greater Flamingo & PM & Phoenicopterus ruber & Phoenicopteridae \\
\hline 19 & Northern Shoveler & PM & Anas clypeata & Anatidae \\
\hline 20 & Black Kite & PM & Milvus migrans & Accipitridae \\
\hline 21 & Arabian Bustard & LM & Ardeotis arabs stieberi & Otididae \\
\hline 22 & Black-winged Stilt & PM & Himantopus himantopus & Recurvirostridae \\
\hline 23 & Greater-painted Snipe & $\mathrm{R}$ & Rostratula benghalensis & Rostratulidae \\
\hline 24 & Spotted Thick-knee & $\mathrm{R}$ & Burhinus capensis & Burhinidae \\
\hline 25 & Senegal Thick-knee & $\mathrm{R}$ & Burhinus senegalensis & Burhinidae \\
\hline 26 & Egyptian Plover & $\mathrm{R}$ & Pluvianus aegyptius & Glareolidae \\
\hline 27 & Spur-winged Plover & PM & Vanellus armatus & Charariidae \\
\hline 28 & Black winged Plover & PM & Vanellus melanopterus & Charariidae \\
\hline 29 & Three-banded Plover & LM & Charadrius tricollaries & Charariidae \\
\hline 30 & Common ringed Plover & PM & Charadrius hiaticula & Charariidae \\
\hline 31 & Little ringed Plover & PM & Charadrius dubius & Charariidae \\
\hline 32 & Kentish Plover & PM & Charadrius alexandrinus & Charariidae \\
\hline 33 & Black-bellied Plover & PM & Pluvialis squatarola & Charariidae \\
\hline 34 & Ruff & PM & Philomachus pugnax & Scolopacidae \\
\hline 35 & Common Sandpiper & PM & Actitis hypoleucos & Scolopacidae \\
\hline 36 & Wood Sandpiper & PM & Tringa glareola & Scolopacidae \\
\hline
\end{tabular}


Cont Table1:

\begin{tabular}{|c|c|c|c|c|}
\hline No & Species Common Names & Status & Scientific Name & Family Name \\
\hline 37 & Green Sandpiper & $\mathrm{PM}$ & Tringa ochropus & Scolopacidae \\
\hline 38 & Terek Sandpiper & PM & Xenus cinereus & Scolopacidae \\
\hline 39 & Common Green Shank & PM & Tringa nebularia & Scolopacidae \\
\hline 40 & Marsh Sandpiper & $\mathrm{PM}$ & Tringa stagnatilis & Scolopacidae \\
\hline 41 & Common Red Shank & $\mathrm{PM}$ & Tringa tetanus & Scolopacidae \\
\hline 42 & Little Stint & PM & Calidaris minuta & Scolopacidae \\
\hline 43 & Red-necked Stint & PM & Calidris ruficollis & Scolopacidae \\
\hline 44 & Sanderling & PM & Calidaris alba & Scolopacidae \\
\hline 45 & Ruddy Turnstone & PM & Arenaria interpres & Scolopacidae \\
\hline 46 & Black-tailed Godwift & PM & Limosa limosa & Scolopacidae \\
\hline 47 & Eurasian Curlew & PM & Numenius arquata & Scolopacidae \\
\hline 48 & Common Snipe & PM & Gallinago gallinago & Scolopacidae \\
\hline 49 & Common Black-headed Gull & PM & Larus ridibundus & Laridae \\
\hline 50 & Lesser-crested Tern & LM & Sterna bengalensis & Laridae \\
\hline 51 & Caspian Tern & $\mathrm{PM}$ & Sterna caspia & Laridae \\
\hline 52 & Gull-billed Tern & PM & Sterna nilotica & Laridae \\
\hline 53 & Common Tern & PM & Sterna hirundo & Laridae \\
\hline 54 & White- winged Tern & PM & Chlidonias leucopterus & Laridae \\
\hline 55 & Whiskerd Tern & PM & Chlidonias hybridus & Laridae \\
\hline 56 & Namaqua Dove & PM & Oena capensis & Columbidae \\
\hline 57 & African mourning Dove & $\mathrm{R}$ & Streptopelia decipiens & Columbidae \\
\hline 58 & Laughing Dove & PM & Sterptopelia senegalensis & Columbidae \\
\hline 59 & Diederik Cuckoo & $\mathrm{R}$ & Chrysococcyx caprius & Cuculidae \\
\hline 60 & Little Swift & PM & Apus affinis & Apodidae \\
\hline 61 & Alpine Swift & PM & Apus melba & Apodidae \\
\hline 62 & African Palm Swift & $\mathrm{R}$ & Cypsiurus parvus & Apodidae \\
\hline 63 & Pied Kingfisher & $\mathrm{R}$ & Ceryl rudis & Alcedinidae \\
\hline 64 & Little Bee-eater & $\mathrm{R}$ & Merops pusillus & Meropidae \\
\hline 65 & Little Green bee-eater & $\mathrm{R}$ & Merops orientalis & Meropidae \\
\hline 66 & African Hoopoe & $\mathrm{R}$ & Upupa africana & Upupidae \\
\hline 67 & Rufous-naped Lark & $\mathrm{R}$ & Mirafra africana & Alaudidae \\
\hline 68 & Crested Lark & $\mathrm{R}$ & Galerida cristata & Alaudidae \\
\hline 69 & Chestnut-backed Sparrow Lark & $\mathrm{R}$ & Eremopterix leucotis & Alaudidae \\
\hline 70 & Common House Martin & PM & Delichon urbica & Hirundinidae \\
\hline 71 & Ethiopian Swallow & $\mathrm{R}$ & Hirundo aethiopica & Hirundinidae \\
\hline 72 & Grey-rumped Swallow & $\mathrm{R}$ & Pseudohirundo griseopyga & Hirundinidae \\
\hline 73 & White Wagtail & PM & Motacilla alba & Motacillidae \\
\hline 74 & Pied Wagtail & $\mathrm{R}$ & Motacilla aguimp & Motacillidae \\
\hline 75 & Yellow Wagtail & PM & Motacilla flava & Motacillidae \\
\hline 76 & Common Bulbul & $\mathrm{R}$ & Pycnonotus barbatus & Pycnonotidae \\
\hline 77 & Isabelline Wheatear & PM & Oenanthe isabellina & Turdidae \\
\hline 78 & Desert Wheatear & PM & Oenenthe deserti & Turdidae \\
\hline 79 & Spotted Morning-thrush & $\mathrm{LM}$ & Cichladusa guttata & Turdidae \\
\hline 80 & Eurasian Golden Oriole 1 & R PM & Oriolus oriolus & Oriolidae \\
\hline 81 & House Sparrow & $\mathrm{R}$ & Passer domesticus & Passeridae \\
\hline 82 & Golden Sparrow & $\mathrm{R}$ & Passer luteus & Passeridae \\
\hline 83 & Village Weaver & $\mathrm{R}$ & Ploceus cucullatus & Ploceidae \\
\hline 84 & Little Weaver & $\mathrm{R}$ & Ploceus luteolus & Ploceidae \\
\hline 85 & Red-billed Quelea & $\mathrm{R}$ & Quelea quelea & Ploceidae \\
\hline 86 & Northern Red Bishop & $\mathrm{R}$ & Euplectes franciscanus & Ploceidae \\
\hline 87 & White-rumped Seed Eater & $\mathrm{R}$ & Serinus leucopygius & Fringillidae \\
\hline
\end{tabular}


The variation in abundance and species composition of birds inhabiting the site during the study period is shown in table (2).

Table 2: Estimates of the numbers of individual birds observed for each species at Sunut forest during the study period (October 2011- June 2012).

\begin{tabular}{|c|c|c|c|c|c|c|c|c|c|c|}
\hline \multirow[b]{2}{*}{ No. } & \multirow[b]{2}{*}{ Species Common Name } & \multicolumn{9}{|c|}{ Estimated numbers of Birds in the study area } \\
\hline & & OCT & NOV & DEC & JAN & FEB & MAR & APR & MAY & JUN \\
\hline 1 & Great White Pelican & 19 & 0 & 0 & 0 & 0 & 38 & 74 & 55 & 57 \\
\hline 2 & Pink Blacked Pelican & 0 & 0 & 0 & 0 & 0 & 0 & 0 & 2 & 3 \\
\hline 3 & Little Grebe & 0 & 0 & 0 & 0 & 0 & 7 & 9 & 0 & 0 \\
\hline 4 & Long-tailed Cormorant & 0 & 0 & 0 & 0 & 0 & 0 & 1 & 3 & 0 \\
\hline 5 & Cattle Egret & 10 & 5 & 3 & 2 & 7 & 6 & 12 & 18 & 20 \\
\hline 6 & Common Saquacco Heron & 2 & 0 & 0 & 0 & 1 & 2 & 5 & 3 & 13 \\
\hline 7 & Little Egret & 25 & 20 & 17 & 7 & 9 & 5 & 15 & 18 & 26 \\
\hline 8 & Great Egret & 3 & 1 & 0 & 0 & 1 & 0 & 3 & 3 & 5 \\
\hline 9 & Golaith Heron & 0 & 0 & 0 & 0 & 0 & 0 & 1 & 0 & 0 \\
\hline 10 & Grey Heron & 3 & 2 & 1 & 0 & 2 & 3 & 1 & 0 & 1 \\
\hline 11 & White Stork & 7 & 0 & 2 & 0 & 0 & 0 & 0 & 0 & 0 \\
\hline 12 & Abdims Stork & 0 & 0 & 0 & 0 & 0 & 0 & 7 & 1 & 2 \\
\hline 13 & Black Stork & 4 & 0 & 0 & 0 & 0 & 0 & 0 & 1 & 1 \\
\hline 14 & Saddle-billed Stork & 0 & 0 & 0 & 0 & 0 & 0 & 0 & 1 & 1 \\
\hline 15 & Sacred Ibis & 3 & 0 & 0 & 0 & 3 & 9 & 10 & 7 & 0 \\
\hline 16 & African Spoonbill & 0 & 0 & 0 & 0 & 0 & 0 & 2 & 4 & 5 \\
\hline 17 & Eurasian Spoonbill & 0 & 0 & 0 & 0 & 0 & 1 & 2 & 3 & 2 \\
\hline 18 & Greater Flamingo & 50 & 55 & 39 & 0 & 0 & 0 & 0 & 0 & 0 \\
\hline 19 & Northern Shoveler & 0 & 0 & 0 & 0 & 5 & 3 & 90 & 5 & 0 \\
\hline 20 & Black Kite & 17 & 15 & 12 & 5 & 4 & 7 & 9 & 4 & 3 \\
\hline 21 & Arabian Bustard & 0 & 0 & 0 & 0 & 0 & 0 & 0 & 0 & 3 \\
\hline 22 & Black-winged Stilt & 7 & 6 & 5 & 6 & 0 & 2 & 3 & 5 & 7 \\
\hline 23 & Greater-painted Snipe & 0 & 0 & 0 & 0 & 0 & 0 & 7 & 0 & 3 \\
\hline 24 & Spotted Thick-knee & 0 & 0 & 0 & 1 & 0 & 0 & 4 & 0 & 1 \\
\hline 25 & Senegal Thick-knee & 0 & 0 & 0 & 0 & 0 & 0 & 0 & 1 & 0 \\
\hline 26 & Egyptian Plover & 0 & 0 & 2 & 4 & 0 & 3 & 15 & 0 & 0 \\
\hline 27 & Spur-winged Plover & 47 & 40 & 45 & 45 & 60 & 65 & 72 & 40 & 43 \\
\hline 28 & Black winged Plover & 0 & 0 & 1 & 4 & 0 & 0 & 0 & 0 & 0 \\
\hline 29 & Three-banded Plover & 0 & 0 & 0 & 0 & 0 & 0 & 0 & 0 & 2 \\
\hline 30 & Common ringed Plover & 5 & 13 & 5 & 6 & 6 & 0 & 9 & 5 & 3 \\
\hline 31 & Little ringed Plover & 0 & 15 & 6 & 4 & 3 & 1 & 3 & 0 & 5 \\
\hline 32 & Kentish Plover & 0 & 0 & 1 & 2 & 2 & 1 & 0 & 1 & 0 \\
\hline 33 & Black-bellied Plover & 0 & 2 & 0 & 0 & 0 & 0 & 0 & 0 & 0 \\
\hline 34 & Ruff & 5 & 0 & 2 & 3 & 1 & 1 & 1 & 0 & 0 \\
\hline 35 & Common Sandpiper & 0 & 7 & 4 & 10 & 15 & 11 & 8 & 1 & 2 \\
\hline 36 & Wood Sandpiper & 0 & 6 & 10 & 7 & 0 & 1 & 1 & 0 & 0 \\
\hline 37 & Green Sandpiper & 0 & 8 & 4 & 5 & 0 & 2 & 1 & 0 & 0 \\
\hline 38 & Terek Sandpiper & 0 & 9 & 3 & 1 & 2 & 1 & 0 & 1 & 1 \\
\hline 39 & Common Green Shank & 0 & 7 & 3 & 8 & 7 & 1 & 1 & 1 & 0 \\
\hline 40 & Marsh Sandpiper & 0 & 0 & 3 & 8 & 10 & 0 & 0 & 0 & 0 \\
\hline 41 & Common Red Shank & 0 & 3 & 3 & 8 & 5 & 1 & 0 & 2 & 0 \\
\hline 42 & Little Stint & 0 & 12 & 5 & 1 & 4 & 3 & 6 & 3 & 0 \\
\hline 43 & Red-necked Stint & 0 & 15 & 5 & 0 & 2 & 0 & 0 & 0 & 0 \\
\hline 44 & Sanderling & 0 & 8 & 9 & 1 & 0 & 9 & 2 & 0 & 0 \\
\hline
\end{tabular}


Cont: Table 2

\begin{tabular}{|c|c|c|c|c|c|c|c|c|c|c|}
\hline \multirow[b]{2}{*}{ No } & \multirow[b]{2}{*}{ Species Common Name } & \multicolumn{9}{|c|}{ Estimated numbers of Birds in the study area } \\
\hline & & OCT & NOV & DEC & JAN & FEB & MAR & APR & MAY & JUN \\
\hline 45 & Ruddy Turnstone & 0 & 0 & 0 & 0 & 0 & 0 & 2 & 0 & 0 \\
\hline 46 & Black-tailed Godwift & 0 & 7 & 20 & 35 & 45 & 15 & 1 & 0 & 0 \\
\hline 47 & Eurasian Curlew & 0 & 0 & 1 & 2 & 7 & 5 & 2 & 0 & 0 \\
\hline 48 & Common Snipe & 3 & 0 & 0 & 0 & 0 & 5 & 7 & 0 & 0 \\
\hline 49 & Common Black-headed Gull & 0 & 0 & 0 & 0 & 0 & 12 & 10 & 0 & 0 \\
\hline 50 & Lesser-crested Tern & 0 & 0 & 0 & 0 & 2 & 5 & 4 & 0 & 0 \\
\hline 51 & Caspian Tern & 0 & 0 & 0 & 0 & 3 & 4 & 1 & 0 & 0 \\
\hline 52 & Gull-billed Tern & 0 & 0 & 0 & 0 & 1 & 3 & 1 & 0 & 0 \\
\hline 53 & Common Tern & 0 & 0 & 0 & 5 & 3 & 8 & 10 & 2 & 0 \\
\hline 54 & White-winged Tern & 0 & 0 & 0 & 0 & 0 & 2 & 8 & 5 & 0 \\
\hline 55 & Whiskerd Tern & 0 & 0 & 0 & 1 & 1 & 5 & 6 & 1 & 0 \\
\hline 56 & Namaqua Dove & 0 & 0 & 0 & 0 & 0 & 1 & 2 & 0 & 1 \\
\hline 57 & African mourning Dove & 9 & 5 & 4 & 8 & 4 & 9 & 7 & 4 & 5 \\
\hline 58 & Laughing Dove & 64 & 45 & 54 & 60 & 66 & 59 & 60 & 63 & 55 \\
\hline 59 & Diederik Cuckoo & 0 & 0 & 0 & 0 & 0 & 1 & 3 & 0 & 0 \\
\hline 60 & Little Swift & 0 & 40 & 48 & 35 & 26 & 0 & 47 & 30 & 0 \\
\hline 61 & Alpine Swift & 0 & 39 & 45 & 50 & 40 & 43 & 45 & 33 & 0 \\
\hline 62 & African Palm Swift & 0 & 0 & 0 & 0 & 0 & 0 & 17 & 0 & 15 \\
\hline 63 & Pied Kingfisher & 2 & 3 & 2 & 6 & 2 & 7 & 4 & 4 & 2 \\
\hline 64 & Little Bee-eater & 0 & 0 & 1 & 0 & 1 & 9 & 1 & 0 & 0 \\
\hline 65 & Little Green bee-eater & 0 & 0 & 0 & 0 & 0 & 1 & 1 & 0 & 0 \\
\hline 66 & African Hoopoe & 0 & 0 & 0 & 0 & 0 & 0 & 1 & 0 & 0 \\
\hline 67 & Rufous-naped Lark & 0 & 0 & 3 & 0 & 1 & 1 & 0 & 0 & 0 \\
\hline 68 & Crested Lark & 5 & 3 & 9 & 7 & 4 & 10 & 10 & 12 & 15 \\
\hline 69 & Chestnut-backed Sparrow & 0 & 0 & 0 & 0 & 0 & 0 & 0 & 93 & 160 \\
\hline 70 & Common House Martin & 0 & 0 & 0 & 0 & 0 & 44 & 88 & 153 & 100 \\
\hline 71 & Ethiopian Swallow & 0 & 66 & 75 & 73 & 76 & 70 & 85 & 59 & 93 \\
\hline 72 & Grey-rumped Swallow & 0 & 0 & 32 & 27 & 12 & 19 & 25 & 0 & 0 \\
\hline 73 & White Wagtail & 15 & 20 & 18 & 13 & 23 & 12 & 1 & 0 & 0 \\
\hline 74 & Pied Wagtail & 0 & 0 & 1 & 5 & 7 & 0 & 3 & 0 & 0 \\
\hline 75 & Yellow Wagtail & 14 & 7 & 10 & 8 & 25 & 2 & 1 & 0 & 0 \\
\hline 76 & Common Bulbul & 0 & 0 & 3 & 6 & 7 & 6 & 3 & 0 & 0 \\
\hline 77 & Isabelline Wheatear & 0 & 2 & 5 & 4 & 2 & 0 & 0 & 0 & 0 \\
\hline 78 & Desert Wheatear & 0 & 0 & 0 & 0 & 0 & 2 & 3 & 0 & 0 \\
\hline 79 & Spotted Morning-thrush & 0 & 0 & 0 & 1 & 0 & 0 & 0 & 0 & 0 \\
\hline 80 & Eurasian Golden Oriole & 0 & 0 & 5 & 0 & 9 & 12 & 0 & 0 & 0 \\
\hline 81 & House Sparrow & 53 & 49 & 47 & 65 & 63 & 72 & 92 & 180 & 1000 \\
\hline 82 & Golden Sparrow & 0 & 0 & 0 & 0 & 0 & 0 & 0 & 93 & 100 \\
\hline 83 & Village Weaver & 0 & 0 & 0 & 0 & 0 & 7 & 45 & 90 & 1000 \\
\hline 84 & Little Weaver & 0 & 0 & 0 & 0 & 0 & 8 & 4 & 83 & 300 \\
\hline 85 & Red-billed Quelea & 0 & 0 & 0 & 0 & 0 & 0 & 0 & 47 & 80 \\
\hline 86 & Northern Red Bishop & 0 & 0 & 0 & 0 & 1 & 2 & 5 & 30 & 900 \\
\hline 87 & White-rumped Seed Eater & 0 & 0 & 0 & 0 & 0 & 0 & 260 & 500 & 600 \\
\hline
\end{tabular}

\section{The diversity of Acacia nilotica-associated Arthropods}

A total of 465 arthropods representing 30 different morphospecies were collected. Collected specimens comprise 2 classes, 10 orders and at least 16 families. Class Insecta was dominant, represented by 7 orders and 16 families while class 
Arachnida was less frequent, represented by only 3 orders. Among insects the dominant orders were Coleoptera (141 individuals), followed by Hemiptera (95 individuals). The least represented orders were Dictyoptera, Lepidoptera and Phasmida. Among Arachnida order Shizomida was dominant, represented by 114 individuals while order Amblypygi density was the bare minimum represented by only 13 individuals. The number of arthropod specimens collected from each order is illustrated in table (3).

Table 3: Density of arthropod orders collected from Acacia nilotica trees at four sites in the Sunut Forest during April-June 2012.

\begin{tabular}{|l|l|l|l|l|l|}
\hline \multirow{2}{*}{ Order } & \multicolumn{5}{|c|}{ Density } \\
\cline { 2 - 6 } & Site 1 & Site 2 & Site 3 & Site 4 & Total \\
\hline Amblypygi & 6 & 2 & 5 & 1 & 14 \\
\hline Araneae & 19 & 7 & 6 & 6 & 38 \\
\hline Coleoptera & 34 & 60 & 36 & 8 & 138 \\
\hline Dictyoptera & 0 & 0 & 1 & 1 & 2 \\
\hline Hemiptera & 49 & 20 & 8 & 18 & 95 \\
\hline Hymenoptera & 7 & 9 & 6 & 3 & 25 \\
\hline Lepidoptera & 1 & 1 & 1 & 0 & 3 \\
\hline Phasmida & 1 & 0 & 1 & 1 & 3 \\
\hline Shizomida & 50 & 26 & 33 & 27 & 136 \\
\hline Thysanoptera & 2 & 4 & 0 & 2 & 8 \\
\hline Total & 175 & 123 & 97 & 67 & 465 \\
\hline
\end{tabular}

Four families of Coleoptera were identified, among them the Scolytidae was the most frequent. Hemipterans were represented by 7 families, from them Miridae was the dominant family. Most of the other orders were represented by only one family. The number of specimens collected from the different families of Coleoptera and Hemiptera are shown in table (4).

Table 4: Density of Coleopteran and Hemipteran families collected from Acacia nilotica trees at four sites in the Sunut Forest during April-June 2012.

\begin{tabular}{|l|l|l|l|l|l|l|}
\hline Order & Family & \multicolumn{6}{|c|}{ Density } \\
\cline { 3 - 7 } & & Site 1 & Site 2 & Site 3 & Site 4 & Total \\
\hline \multirow{5}{*}{ Coleoptera } & Coccinellidae & 5 & 10 & 4 & 3 & 22 \\
\cline { 2 - 7 } & Chrysomelidae & 3 & 11 & 0 & 2 & 16 \\
\cline { 2 - 7 } & Curculionidae & 15 & 2 & 4 & 0 & 21 \\
\cline { 2 - 7 } & Scolytidae & 14 & 37 & 28 & 3 & 82 \\
\cline { 2 - 7 } & Pentatomidae & 0 & 2 & 3 & 3 & 8 \\
\cline { 2 - 7 } & Miridae & 40 & 15 & 4 & 15 & 74 \\
\cline { 2 - 7 } & Lygaeidae & 2 & 1 & 0 & 0 & 3 \\
\cline { 2 - 7 } & Coreidae & 2 & 0 & 0 & 0 & 2 \\
\cline { 2 - 7 } & Coccidae & 1 & 1 & 1 & 0 & 3 \\
\cline { 2 - 7 } & Fulgoridae & 2 & 1 & 0 & 0 & 3 \\
\cline { 2 - 7 } & Aphididae & 2 & 0 & 0 & 0 & 2 \\
\hline
\end{tabular}

\section{The diversity of ground arthropods}

A total of 1286 specimens of ground arthropods, representing three classes, 11 orders and 18 morphospecies were collected (Table 5). The class Arachnida represents $14 \%$ of the total number of specimens collected. These included four morphospecies, while the class Diplopoda was represented by only 3 individuals $(0.23 \%)$. Insecta was the dominant class, comprising $86 \%$ of specimens collected, including 9 orders and 13 distinct morphospecies. Among the insects Hymenoptera 
was the most represented order (44\%), followed by Coleoptera (25\%) and Orthoptera $(9 \%)$. The rest of insect orders represent only $8 \%$ of the total collection.

Table 5: Density of the different orders of ground arthropods collected at two sites in the Sunut Forest during February 2012.

\begin{tabular}{|l|l|l|l|l|l|}
\hline \multicolumn{2}{|l}{} & Site A & Site B & Total Number & Percentage (\%) \\
\hline Arachnida & & 150 & 24 & 174 & 13.53 \\
\hline Diplopoda & Coleoptera & 1 & 2 & 3 & 0.23 \\
\hline \multirow{5}{*}{ Insecta } & 141 & 176 & 317 & 24.65 \\
\cline { 2 - 6 } & Hymenoptera & 22 & 545 & 567 & 44.09 \\
\cline { 2 - 6 } & Orthoptera & 69 & 46 & 115 & 8.94 \\
\cline { 2 - 6 } & Dermaptera & 22 & 0 & 22 & 1.71 \\
\cline { 2 - 6 } & Dictyoptera & 0 & 2 & 2 & 0.16 \\
\cline { 2 - 6 } & Collembola & 4 & 0 & 4 & 0.31 \\
\cline { 2 - 6 } & Hemiptera & 33 & 6 & 39 & 3.03 \\
\cline { 2 - 6 } & Siphnoptera & 0 & 3 & 3 & 0.23 \\
\cline { 2 - 6 } & Mallophaga & 0 & 1 & 1 & 0.08 \\
\hline
\end{tabular}

\section{DISCUSSION}

During this study, a preliminary survey of biological diversity has been conducted at Sunut Forest. Three groups were targeted during the survey: birds, treeassociated arthropods and ground arthropods. The goal of the investigation was to provide basic data that can be used for future management and conservation activities at the forest. Overall, the study demonstrates that this site represents a hot spot for avian and arthropod diversity, as both the density and species diversity recorded there were higher than the surrounding, semi desert region of Khartoum. These findings support the view of Sudanese conservationists that Sunut forest is a valuable and unique site that should be maintained as close as possible to its natural state and protected from the severe human impact in the vicinity.

Despite the limited spatial and temporal scale of the study, 87 bird species were recorded at Sunut forest, thus demonstrating the special richness of the site with birds and, consequently, its touristic value for bird watching activities. The avifauna at Sunut forest was found to show considerable temporal fluctuations with respect to both abundance and species diversity. These variations can be attributed to two main factors (e.g. Pearson \& Backhurst, 1976; Mac Nally, 1996; Yahner, 1997): first, the seasonal change in water availability that is associated with the Nile flood, this change influences both vegetation cover and insect density at the forest, and thus affects the attractiveness of the site for birds. The second factor which underlies the temporal variations is bird migrational patterns, as most of the recorded bird species (i.e. 50) were Palaearctic migrants. The annual movements of migrant birds to and from the site are likely to cause substantial temporal changes in the avifauna composition.

An outstanding feature of Acacia nilotica is its tolerance to prolonged periods of flood and inundation, which enables the species to flourish in periodically flooded habitats such as Sunut forest (Booth, 1966). In this study, the association between Acacia nilotica and arthropods was investigated. Arthropods were found to show higher density and diversity at A. nilotica trees than at the surrounding semi-desert Khartoum region. However, both the density and diversity appear to be somehow lower than those recorded from similar habitats (Cloudsely-Thompson, 1964; Elobeid, 1990). This may refer to the fact that the sampling period was restricted to the dry season only. However, this may also suggest a poor association of 
invertebrates with A. nilotica trees. Indeed, some studies have reported that Sunut tress have powerful algaecidal activity due to their high tannin content. Aqueous extracts of the plant ripe pods also showed molluscicidal activity against snail vectors of Schistosomiasis (Ayoub, 1982). Such properties suggest similar effects on insects and other invertebrates, thus explaining the relatively poor density and diversity recorded in the current study. However, such conclusion could only be confirmed through conducting studies that compare invertebrate communities associated with $A$. nilotica to those associated with other tree species co-existing in the same habitat such as A. seyal, A. albida, Balanitus egyptiaca, Tamarax nilotica, Ziziphus spinachrist.

The structure of ground arthropod community is affected by several factors such as seasonal changes, habitat structure and land use (McIntyre et al., 2001). During the current investigation of ground arthropods at Sunut forest, considerable species diversity was recorded (mostly of insects). Interestingly, no clear variation was observed in ground arthropods community between the cultivated area and the forest area, despite the marked differences in the soil environment and the intensity of human influence. The observed homogeneity might result from the spatial distribution of the two sampling sites, as they were rather close to each other. The sampling site at the forest was close to the forest edge where the human impact is higher than the inner parts of the forest, due to the extensive disturbance caused by recreation activities.

The current research could be considered as pilot one towards investigation of the biodiversity at Sunut forest. Future research should adopt larger scale of sampling, both spatially and temporally; species density and diversity should be investigated during different seasons as well as from different sites in the forest. More precise taxonomical methodologies, aiming to provide identification at the species level, should be applied during surveys of arthropod diversity at the site. In addition, the diversity at the areas surrounding Sunut forest should be assessed as well, so that conclusions concerning the biodiversity status of the forest can be made on comparative basis. The ecological impact of construction, recreation and waste disposal activities on the site should also be targeted by future research.

\section{ACKNOWLEDGMENTS}

The authors wish to thank Dr. Ahmed Salah Khalil and Dr. Sami Saeed for their important contribution to this work.

\section{REFERENCES}

Abd-Alrahman, S. A. (1998). Birds of Sunut Forest. M. Sc. Thesis. Zoology Department, Faculty of Science, University of Khartoum.

Abushama, H. M. (1994). The ecological impact of liquid waste disposal on biological communities in a pool at Khartoum Sunut Forest. B. Sc. Hons. Dissertation. Zoology Department, Faculty of Science, University of Khartoum.

Ahmed, H. M. (1998). The auto ecology of Acacia nilotica, case study Khartoum Sunut Forest. M. Sc. Thesis. Botany Department, Faculty of Science, University of Khartoum.

Altayeb, A. M and Hamed, D. M. (2003). Information Sheet on the Ramsar Wetlands (RIS). 
Ayoub, S. M. H. (1982). Algaecidal properties of Acacia nilotica plant. Medica, 35: 157-177.

Booth, G. A. (1966). Savanna forestation in the Sudan. Sudan Silva, 11: 15-21.

Cloudsely-Thompson, J. L. (1964). Guide to desert invertebrates of Khartoum Province. Sudan Notes and Records, 45: 127-136.

Elobeid, M. A. (1990). The invertebrate fauna of four selected islands in Khartoum Province. B. Sc. Hons. Dissertation. Zoology Department, Faculty of Science, University of Khartoum.

Lado, B. D. (1994) Birds of Sunut forest in Khartoum. M. Sc. Thesis. Zoology Department, Faculty of Science, University of Khartoum.

Mac Nally R. (1996). A winter's tale: Among-year variation in bird community structure in a southeastern Australian forest. Australian Journal of Ecology, 21: 28091.

McIntyre, N. E., Rango, J., Fagan, W. F. and Faeth, S. H. (2001). Ground arthropod community structure in a heterogeneous urban environment. Landscape and Urban Planning, 52: 257-274.

Mohamed, A. O. (1986). An ecological study on the regeneration of Acacia nilotica in the Sunut Forest, Khartoum. B. Sc. Hons. Dissertation. Botany Department, Faculty of Science, University of Khartoum.

Nikolaus, G. (1987). Distribution atlas of Sudan's birds with notes on habitat and status. Bonner zoologische monographien, Bonn. No. 25. 322pp.

Pearson, D. J. and Backhurst, G.C. (1976). The southward migration of palearctic birds over Ngulia, Kenya. Ibis, 118: 78-105.

Shawki and Musnad (1964). Guide to Khartoum Arboretum and forest nursery. Forest Department Bulletin, 9: 16-22.

Stevenson, T. and Fanshawe, J. (2002). Birds of East Africa. Christopher Helm, London.

Yahner R. H. (1997). Long-term dynamics of bird communities in a man-aged forested landscape. Wilson Bulletin, 109: 595-613. 